\title{
An enhanced RRM2 siRNA delivery to rheumatoid arthritis fibroblast-like synoviocytes through a liposome-protamine-DNA-siRNA complex with cell permeable peptides
}

\author{
XIN WANG $^{1}$, XUEPING WANG $^{2}$, JIN SUN $^{3}$ and SHIOU FU ${ }^{2}$ \\ ${ }^{1}$ The First Department of Pain Management and ${ }^{2}$ The Second Department of Pain Management, \\ Qingdao Municipal Hospital, Qingdao, Shandong 266011; ${ }^{3}$ International Joint Cancer Institute, \\ Second Military Medical University, Shanghai 200433, P.R. China
}

Received March 26, 2018; Accepted August 6, 2018

DOI: $10.3892 /$ ijmm.2018.3815

\begin{abstract}
Rheumatoid arthritis (RA) is considered to be a systemic autoimmune disease that induces systemic complications and progressive disability. It affects a large number of people. RA fibroblast-like synoviocytes (RA-FLS) promote the progression of RA through the secretion of proinflammatory cytokines and increasing invasiveness into the extracellular matrix. Therefore, targeting RA-FLS represents a potential approach for the treatment of RA. Ribonucleotide reductase M2 (RRM2), a critical protein for DNA synthesis and repair, may promote the proliferation of cells and inhibit cellular apoptosis. In previous studies it has been confirmed that the suppression of RRM2 markedly suppressed the proliferation of liver cancer cells. In the present study, a cell permeable peptide-conjugated liposome-polycation-DNA (LPD) complex loaded with RRM2 small interfering RNA (siRNA) (CCP-LPDR) was developed, aiming to increase the levels of apoptosis and inhibit the proliferation of RA-FLS. CCP-LPDR is a small-sized molecule $(\sim 130 \mathrm{~nm})$ with high encapsulation efficiency of siRNA ( $>90 \%)$ and high stability. Furthermore, it was verified that CCP-LPDR markedly suppressed RRM2 gene and protein expression by $\sim 80 \%$. Notably, CCP-LPDR efficiently targeted RA-FLS, resulting in a marked decrease in the proliferation and increase in the level of apoptosis in RA-FLS. In addition, the levels of proinflammatory cytokines tumor necrosis factor- $\alpha$ and interleukin- 6 were markedly decreased in RA-FLS following CCP-LPDR
\end{abstract}

Correspondence to: $\mathrm{Dr}$ Shiou Fu, The Second Department of Pain, Qingdao Municipal Hospital, 1 Jiaozhou Road, Qingdao, Shandong 266011, P.R. China

E-mail: bhyxouou@163.com

Key words: ribonucleotide reductase $\mathrm{M} 2$, fibroblast-like synoviocytes, small interfering RNA, liposomes, rheumatoid arthritis treatment. Therefore, CCP-LPDR may efficiently deliver RRM2 to RA-FLS and represent a potential treatment for RA.

\section{Introduction}

The prevalence of rheumatoid arthritis (RA), a systemic autoimmune disease that induces systemic complications and progressive disability, is rising steadily $(1,2)$. Generally, the prevalence of RA worldwide is $\sim 1 \%$ of the total adult population $(1,2)$. The characteristics of RA include progressive joint destruction and disability, and it is associated with high prevalence rates of comorbidities including cardiovascular diseases and other extra-articular and systemic diseases (3). Compared with the normal population, a decreased life expectancy in patients with RA has been demonstrated (4). Therefore, the development of an effective treatment for RA is critical for the improvement of the health of these patients. The aims of the treatments of RA include the suppression of inflammation, remission of severe symptoms, prevention of organ and joint damage and improvement of physical function $(4,5)$. However, RA treatment is costly with respect to the surgical procedures, medications including biologics and indirect costs (5). For example, at present the approaches for the treatment of RA include the use of anti-rheumatic drugs, but a good response has only been observed in a minority of patients (5). Therefore, it is necessary to optimize the treatment strategies to ameliorate the clinical and socioeconomic effects of RA.

Although the exact immunological mechanism of RA remains unclear, genetic and environmental factors have been demonstrated to contribute to the pathogenesis of RA $(6,7)$. Several previous studies have suggested that RA fibroblast-like synoviocytes (RA-FLS) serve a vital role in RA development by various mechanisms (6-8). Firstly, RA-FLS produce proinflammatory cytokines, including interleukin-6 (IL-6) and tumor necrosis factor- $\alpha$ (TNF- $\alpha$ ), achieving a perpetual state of inflammation and cartilage destruction (1-3). Secondly, as a uniquely aggressive phenotype, RA-FLS exhibit significant levels of invasiveness into the extracellular matrix and exacerbate joint damage (8). The excessively proliferated RA-FLS 
increase oxygen consumption and hypoxia in the local microenvironment, resulting in a series of events including proinflammatory cell infiltration, synovium inflammation and degradation of cartilage (9). These processes occur simultaneously, and develop a positive feedback loop, resulting in the promotion of RA progression $(9,10)$. Therefore, targeting RA-FLS may improve the clinical outcomes of RA treatment, and the induction of proliferation inhibition and apoptosis of RA-FLS represents a promising approach in the treatment of RA $(8,10)$.

Ribonucleotide reductase M2 (RRM2) is a crucial protein that regulates the formation of deoxyribonucleotides; therefore, it is important for the repair and synthesis of DNA (11). Inhibition of RRM2 significantly inhibits cellular proliferation and induces cell apoptosis $(12,13)$. A previous study suggested that changes in RRM2 expression levels may exhibit a marked effect on tumor metastasis and progression, making it a promising cancer therapeutic target (14). Gemcitabine and GTI-2040 are 2 RRM2 inhibitors and have undergone a clinical trial for cancer therapy (trial no. NCT00078962) (13). Gao et al (14) confirmed that the expression of RRM2 has been identified to be significantly increased in liver cancer compared with in non-liver cancer tissue, and RRM2 suppression may significantly inhibit the proliferation and migration of liver cancer cells, suggesting that RRM2 is a promising target in the therapy of liver cancer. As RRM2 suppression has been validated to decrease the levels of cellular proliferation and induce cellular apoptosis (14), we hypothesized that the inhibited expression of RRM2 in RA-FLS may markedly suppress the proliferation and increase apoptosis in RA-FLS. Nevertheless, to the best of our knowledge, there has been limited data on the effect of RRM2 suppression in RA until now.

Genetic therapy, also termed gene therapy, which is the removal or alteration of genes in cells for therapeutic purposes, represents a promising method for treating various diseases, including RA $(15,16)$. For the treatment of RA, the gene therapy approach may confer potential effects through the specific delivery of various gene products. At present, a number of studies have been performed to investigate the therapeutic efficacy of gene therapy in animal models $(15,16)$. Tomita et al $(16)$ demonstrated that the treatment of rats with collagen-induced arthritis with nuclear factor kappa-light-chain-enhancer of activated B cells decoy oligodeoxynucleotides-loaded liposomes led to an amelioration of arthritis. Notably, gene therapy using small interfering RNA (siRNA) represents an elegant approach with great promise for the treatment of various diseases, and is also a promising alternative to chemotherapy, which is associated with several side effects (17-20). As siRNA molecules alone cannot cross cellular barriers to reach the targets inside the cells, formulations based on nanoparticles have been demonstrated to significantly increase the cellular delivery of siRNA and facilitate siRNA-based therapy in clinical settings (17-20). In previous studies, we successfully constructed a targeted liposome-polycation-DNA complex (LPD) conjugated with the epidermal growth factor receptor antibody to deliver siRNA efficiently to cancer cells and observed marked gene silencing activity and anticancer efficacy (17-19).

Cell permeable peptides (CPP), also termed protein transduction domains, are a diverse class of peptides with dozens of amino acids that enhance the cellular uptake of various substances including nanoparticles $(21,22)$. CyLoP-1 is a cationic CPP rich in cysteine and has been demonstrated by Jha et al (23) to exhibit the efficient cellular delivery of various agents. In order to efficiently deliver RRM2 siRNA to RA-FLS and inhibit their proliferation, the present study developed a cell permeable peptide-conjugated liposome/protamine/DNA/RRM2 siRNA complex (CCP-LPDR) as a nanoparticle-based drug delivery system for RA-FLS. We hypothesize that CCP-LPDR may deliver RRM2 siRNA to RA-FLS effectively; achieving increased therapeutic effect in RA-FLS. The in vitro targeting, gene silencing activity and cellular apoptosis-inducing activity of CCP-LPDR in RA-FLS were then examined by the flow cytometry and reverse transcription quantitative polymerase chain reaction (RT-qPCR). The present study demonstrated that CCP-LPDR efficiently delivered RRM2 siRNA to RA-FLS and achieved an improved therapeutic efficacy against RA-FLS compared with the non-targeted control.

\section{Materials and methods}

Reagents. Grade X protamine, calf thymus DNA and cholesterol were purchased from Sigma-Aldrich; Merck KGaA (Darmstadt, Germany). The following reagents were purchased from Avanti Polar Lipids (Alabaster, AL, USA): 1,2-dioleoyl-3-trimethylammonium-propane (DOTAP, a cationic lipid); functional PEGylated lipids with a maleimide group, 1,2-distearoyl-sn-glycero-3-phosphoethanolamine-N-[maleimide (polyethylene glycol)-2000] (DSPE-PEG-Mal); and, 1,2-dioleoyl-sn-glycero-3-phosphoethanolamine-N-carboxyfluorescein (CFPE, a fluorescent lipid). GL Biochem (Shanghai) Ltd., (Shanghai, China) synthesized the cell permeable peptides, CRWRWKCCKK (CyLoP-1 CCP). Shanghai GenePharma Co. Ltd. (Shanghai, China) synthesized and provided the following siRNAs: Negative control (NC) siRNA, fluorescent siRNA (FAM-siRNA) and RRM2 siRNA. The sequences of siRNAs are summarized in Table I. Dojindo Molecular Technologies, Inc. (Kumamoto, Japan) provided the cellular proliferation kit Cell Counting Kit-8 (CCK-8), and the Reverse Transcription System kit was provided by Promega Corporation (Madison, WI, USA). Thermo Fisher Scientific, Inc. (Waltham, MA, USA) provided the TRIzol ${ }^{\circledR}$ reagent and $S Y B R^{\mathrm{TM}}$-Green PCR Master Mix. R\&D Systems, Inc. (Minneapolis, MN, USA) provided the ELISA kits to measure the concentration of TNF- $\alpha$ (cat. no. DTA00C) and IL-6 (cat. no. D6050). Ultra-4 centrifugal filter devices were purchased from EMD Millipore (Billerica, MA, USA).

\section{Cell culture of human RA-FLS. The RIKEN BioResource} Center (Tsukuba, Japan) provided the human RA-FLS MH7A cell line. The cells were maintained in $10 \mathrm{~cm}$ cultured dishes at $37^{\circ} \mathrm{C}$ in an incubator with a humidified atmosphere of $5 \% \mathrm{CO}_{2}$. The medium used for cell culture was Dulbecco's modified Eagle's medium with $10 \%$ fetal bovine serum (Thermo Fisher Scientific, Inc.). Cells in the exponential phase of growth were used for subsequent experiments.

Fabrication of LPD complex. The development of liposomes was performed using a protocol as previously 
Table I. qPCR primers and siRNA.

A, qPCR primer sequences

\begin{tabular}{llll}
\hline Gene name & \multicolumn{1}{c}{ Forward (5'-3') } & Reverse (5'-3') & Product size, bp \\
\hline$\beta$-actin & CGTGGACATCCGTAAAGACC & ACATCTGCTGGAAGGTGGAC & 209 \\
RRM2 & TCTATGGCTTCCAAATTGCC & GACACAAGGCATCGTTTCAA & 128 \\
TNF- $\alpha$ & CACCACTTCGAAACCTGGGA & TGTAGGCCCCAGTGAGTTCT & 105 \\
IL-6 & CTCAATATTAGAGTCTCAACCCCCA & GAGAAGGCAACTGGACCGAA & 163 \\
\hline
\end{tabular}

$\mathrm{B}$, siRNA sequences

\begin{tabular}{llll}
\hline Gene name & Forward (5'-3') & Reverse (5'-3') & Product size, bp \\
\hline NC & UUCUCCGAACGUGUCACGUTT & ACGUGACACGUUCGGAGAATT \\
RRM2 & GAUUUAGCCAAGAAGUUCAGA & UGAACUUCUUGGCUAAAUCGC & - \\
\hline
\end{tabular}

qPCR, Quantitative polymerase chain reaction; siRNA, small interfering RNA; RRM2, ribonucleotide reductase M2; TNF- $\alpha$, tumor necrosis factor $\alpha$; IL-6, interleukin-6; NC, negative control.

described (Fig. 1) $(11,15,16)$. Briefly, cationic liposomes (the concentration of liposomes was $10 \mathrm{mM}$ with a $1: 1$ molar ratio of DOTAP and cholesterol) were developed by a thin film method based on hydration. The fluorescent liposomes with CFPE was constructed by initially adding 1\% CFPE (molar ratio) to the lipid mixture composed of cholesterol and DOTAP. Multi-layer liposomes (MLL) were formed following the hydration of the lipid film with deionized water. Subsequent to hydration, MLL were then extruded to form single-layer liposomes composed of DOTAP/cholesterol. To prepare the LPD, the initially prepared DOTAP/cholesterol liposomes (125 $\mu \mathrm{l})$ were mixed with $1 \mathrm{mg} / \mathrm{ml}$ protamine $(30 \mu \mathrm{l})$ to produce 'solution I'. At total of $24 \mu \mathrm{g}$ calf thymus DNA and $24 \mu \mathrm{g}$ siRNA $(20 \mu \mathrm{M})$ were also mixed together to form 'solution II'. To form LPD, solutions I and II were then mixed together. At $50^{\circ} \mathrm{C}$, the LPD complex was mixed with $40 \mu 1$ DSPE-PEG-Mal micelles (10 $\mathrm{mg} / \mathrm{ml})$. For peptide conjugation, $80 \mu \mathrm{g} \mathrm{CCP}$ was mixed with the DSPE-PEG-Mal-modified LPD complex at $25^{\circ} \mathrm{C}$ for $6 \mathrm{~h}$.

Similar to CCP-LPDR, non-targeted LPD complexes without $\mathrm{CCP}$ were developed without conjugation of any peptides. The abbreviations used were follows: CCP-LPDR; LPDR; CCP-conjugated LPD complex loaded with negative control siRNA (CCP-LPDN); and LPD complex loaded with negative control siRNA (LPDN).

Size, encapsulation efficacy and stability of cationic liposomes. The cationic liposomes $(10 \mu \mathrm{l})$ were diluted with $1 \mathrm{ml}$ deionized water, and the characteristics, including size, polydispersity index (PDI) and zeta $(\zeta)$ potential of the liposomes were determined with the Zeta sizer Nano S (Malvern Instruments, Ltd., Malvern, UK). The siRNA encapsulation efficiency of the liposomes was examined by an ultrafiltration-based approach: Following the addition of the FAM-siRNA-loaded liposomes in the Ultra- 4 centrifugal filter devices, the centrifugation of the filter devices was performed for $30 \mathrm{~min}$ at 3,500 $\mathrm{x} \mathrm{g}$ and $25^{\circ} \mathrm{C}$ to remove the unloaded siRNA. Following centrifugation, deionized water was added. Subsequent to repeated ultrafiltration four times, the unloaded FAM-siRNA were collected and quantified using the calibration curve of FAM-siRNA with Microsoft Excel 2010 (Microsoft Corporation, Redmond, WA, USA). The fluorescence for FAM-siRNA was examined with the Synergy ${ }^{\mathrm{TM}} 4$ (Biotek Instruments, Inc., Winooski, VT, USA) at an excitation wavelength of $495 \mathrm{~nm}$ and emission wavelength of $525 \mathrm{~nm}$. The siRNA encapsulation efficiency, expressed as percentage, was calculated using the following formula $=[($ The mass of total siRNA - the mass of unloaded siRNA)/the mass of total siRNA] x 100 .

The evaluation of the stability of the liposomes was performed as follows: Firstly, the liposomes were suspended in various DMEM media, including PBS, $10 \%$ FBS or PBS with $20 \% \mathrm{FBS}$. Then the solution was incubated at $25^{\circ} \mathrm{C}$ for 5 days. Each day, an aliquot of liposomes was removed for the analysis of the change in size of the cationic liposomes. An aliquot of liposomes $(10 \mu \mathrm{l})$ were diluted with $1 \mathrm{ml}$ deionized water and the sizes of the liposomes were determined with the Zetasizer Nano S (Malvern Instruments, Ltd.) using the in-built software.

In vitro binding of liposomes with RA-FLS. RA-FLS were plated in 48-well plates overnight. The density of RA-FLS was $6 \times 10^{4}$ cells/well. Following this, the old medium was discarded, and fresh medium was added to the cell culture plates. The fresh medium contained the fluorescent liposomes (FAM-siRNA loaded liposomes: Final FAM-siRNA concentration, $200 \mathrm{nM}$; CFPE labeled liposomes: Final CFPE concentration, $20 \mathrm{ng} / \mathrm{ml}$ ). Subsequent to treatment with liposomes for different lengths of times (1, 2, 4, 6, 12, 18 and $24 \mathrm{~h}$ ), the cells were washed with PBS, trypsinized, washed again, suspended in PBS $(0.3 \mathrm{ml})$ and examined using a flow cytometer (BD Biosciences, San Jose, CA, USA). Data was analyzed using FlowJo (version 10; FlowJo LLC, Ashland, OR, USA). 
$R T-q P C R$. Using TRIzol ${ }^{\circledR}$ reagent (Thermo Fisher Scientific, Inc.), the extraction of RNA from the RA-FLS was performed following the manufacturer's protocol. The synthesis of the first-strand cDNA was performed with the Reverse Transcription System kit according to the manufacturer's protocol. The PCR was performed with $\mathrm{SYBR}^{\mathrm{TM}}$ Green PCR Master Mix in a Roche Light Cycler (Roche Diagnostics $\mathrm{GmbH}$, Mannheim, Germany). The PCR thermocycler conditions were described as follows: Denaturation for $2 \mathrm{~min}$ at $95^{\circ} \mathrm{C}$, then 40 cycles of denaturation at $95^{\circ} \mathrm{C}$ for $3 \mathrm{sec}$, annealing at $55^{\circ} \mathrm{C}$ for $10 \mathrm{sec}$ and extension at $72^{\circ} \mathrm{C}$ for $25 \mathrm{sec}$. The $2^{\Delta \Delta \mathrm{Cq}}$ method was used to quantify the expression of Mrna (24). The sequences of primers used for $\beta$-actin, RRM2, TNF- $\alpha$ and IL-6 are summarized in Table I.

Western blot analysis. The extraction of proteins from the cells was performed using radioimmunoprecipitation assay lysis buffer (Beyotime Institute of Biotechnology, Haimen, China). The protein concentration was determined by the Pierce BCA Protein Assay kit (Thermo Fisher Scientific, Inc.). Each lane of $10 \%$ SDS-PAGE was loaded with $50 \mu \mathrm{g}$ protein. Proteins were then transferred onto polyvinylidene fluoride membranes. The membranes were blocked with $10 \%$ bovine serum albumin (Thermo Fisher Scientific, Inc.) at $95^{\circ} \mathrm{C}$ overnight. Following the transfer of the proteins to the membrane, the following antibodies were added to the membrane: The primary antibody was the mouse anti-human RRM $2 \mathrm{mAb}$ (1:1,000 dilution; cat. no. ab57653; Abcam, Cambridge, MA, USA). The secondary antibody was the horseradish peroxidase-conjugated goat anti-mouse IgG (1:1,500 dilution; ab97023; Abcam). The control antibody was GAPDH (cat. no. ab9484; 1:1,000 dilution; Abcam). The visualization of the bands was performed by the Amersham ${ }^{\mathrm{TM}}$ ECL Plus ${ }^{\mathrm{TM}}$ kit (GE Healthcare, Chicago, IL, USA) and the Bio-Rad ChemiDoc XRS system with Quantity One 1-D analysis software (version 4.6.8; Bio-Rad Laboratories, Inc., Hercules, CA, USA).

Cell viability and apoptosis assays. The cell viability of RA-FLS following treatment with liposomes was examined using a CCK-8 assay. RA-FLS were plated for $16 \mathrm{~h}$ in 96 -well cell culture plates. The density of cells was $1 \times 10^{4}$ cells/well. Following incubation overnight at $37^{\circ} \mathrm{C}$, the old medium was removed, and the cells were exposed to fresh medium containing liposomes (200 $\mathrm{nM}$ siRNA) for $6 \mathrm{~h}$ at $37^{\circ} \mathrm{C}$. Following this treatment, the drugs were discarded, and medium was replaced by fresh medium. A total of $60 \mathrm{~h}$ later, the cell viability of RA-FLS was measured following the protocol of the manufacturer of the CCK-8 kit. The cell apoptosis assay was performed using the Alexa Fluor ${ }^{\circledR} 488$ Annexin V Apoptosis kit (Thermo Fisher Scientific, Inc.), following the manufacturer's protocol. RA-FLS were plated in 12-well cell culture plates overnight. The density of RA-FLS was $2 \times 10^{5}$ cells/well. Following incubation at $37^{\circ} \mathrm{C}$, the cells were then treated with liposomes (200 nM siRNA) for $6 \mathrm{~h}$ at $37^{\circ} \mathrm{C}$. After $6 \mathrm{~h}$, the drugs were removed, and medium was replaced. A total of $60 \mathrm{~h}$ later, the cells were collected using trypsinization and washed twice. Subsequent to washing, the collected cells were re-suspended in an annexin-binding buffer. Then, $5 \mu 1$ propidium iodide (PI) and $5 \mu$ l Alexa Fluor ${ }^{\circledR} 488$ Annexin V were added. After
$15 \mathrm{~min}, 400 \mu \mathrm{l}$ annexin-binding buffer was added to the cells. The analysis of the cells was performed with a flow cytometer (BD Biosciences). Data was analyzed using FlowJo software (version 10).

Analysis of proinflammatory cytokines in RA-FLS following liposome treatment. RA-FLS were plated in 12-well cell culture plates overnight. The density was $2 \times 10^{5}$ cells/well. Then, the RA-FLS were treated with liposomes $(200 \mathrm{nM}$ siRNA) for $6 \mathrm{~h}$. The drugs were removed $6 \mathrm{~h}$ later, and medium was replaced. A total of $60 \mathrm{~h}$ later, the protein and mRNA levels of cytokines were measured using the IL- 6 and TNF- $\alpha$ ELISA kits and RT-qPCR, respectively. The samples (200 $\mu \mathrm{l})$ were added to the ELISA plates and incubated for $2 \mathrm{~h}$ at $37^{\circ} \mathrm{C}$. The sample was then aspirated and washed with PBS. Then, $200 \mu 1$ conjugate was added to the plate and incubated for $4 \mathrm{~h}$ at $37^{\circ} \mathrm{C}$. The substrate solution was then added, followed by the stop solution. Finally, the absorbance of the wells at $450 / 540 \mathrm{~nm}$ was examined with a microplate reader (Biotech ELx800 Universal; BioTek Instruments, Inc.). The protein and mRNA levels of cytokines were expressed as the percentage of the protein and mRNA levels of the treated groups normalized to the untreated group.

Statistical analysis. The data was analyzed using SPSS (version 13; SPSS, Inc., Chicago, IL, USA). A Student's unpaired t-test was used to examine the differences between two groups, and a one-way analysis of variance with a Student-Newman-Keuls post hoc test was performed to examine the differences among $\geq$ three groups. $\mathrm{P}<0.05$ was considered to indicate a statistically significant difference. Unless otherwise stated, all data in the present study are presented as the mean \pm standard deviation.

\section{Results}

Production and properties of the prepared liposomes. Fig. 1 indicates that the DOTAP/cholesterol liposomes were synthesized by hydrating a lipid-film composed of DOTAP and cholesterol. Then, the DOTAP/cholesterol liposomes were conjugated with RRM2 siRNA, calf thymus DNA and protamine to form LPDR. The LPDR were then modified with DSPE-PEG-Mal to prolong their circulation times. The grafted PEG of DSPE-PEG-Mal was able to cover the surface of liposomes efficiently and prevent the opsonization of liposomes. The CCP were conjugated to the DSPE-PEG-Mal-modified LPDR to construct CCP-LPDR, to increase the uptake of liposomes in RA-FLS.

The characteristics, including size, distribution, $\zeta$ potential, polydispersity index (PDI) and encapsulation efficiency of liposomes are summarized in Table II. LPDR and LPDN were $\sim 100 \mathrm{~nm}$ in size. Conjugation with CPP did not have a significant effect on the liposome size, as observed by the similar sizes of LPDR and CCP-LPDR, and those of LPDN and CCP-LPDN. The low PDI $(<0.2)$ of all the liposomes indicated that the prepared liposomes had good homogeneity. All the liposomes exhibited positive $\zeta$ potential of $\sim 20 \mathrm{mV}$. The siRNA encapsulation efficiency of LPD, CCP-LPDN and CCP-LPDR was $>90 \%$ (Table II), indicating that the prepared LPD complex was an efficient drug delivery system for siRNA. 


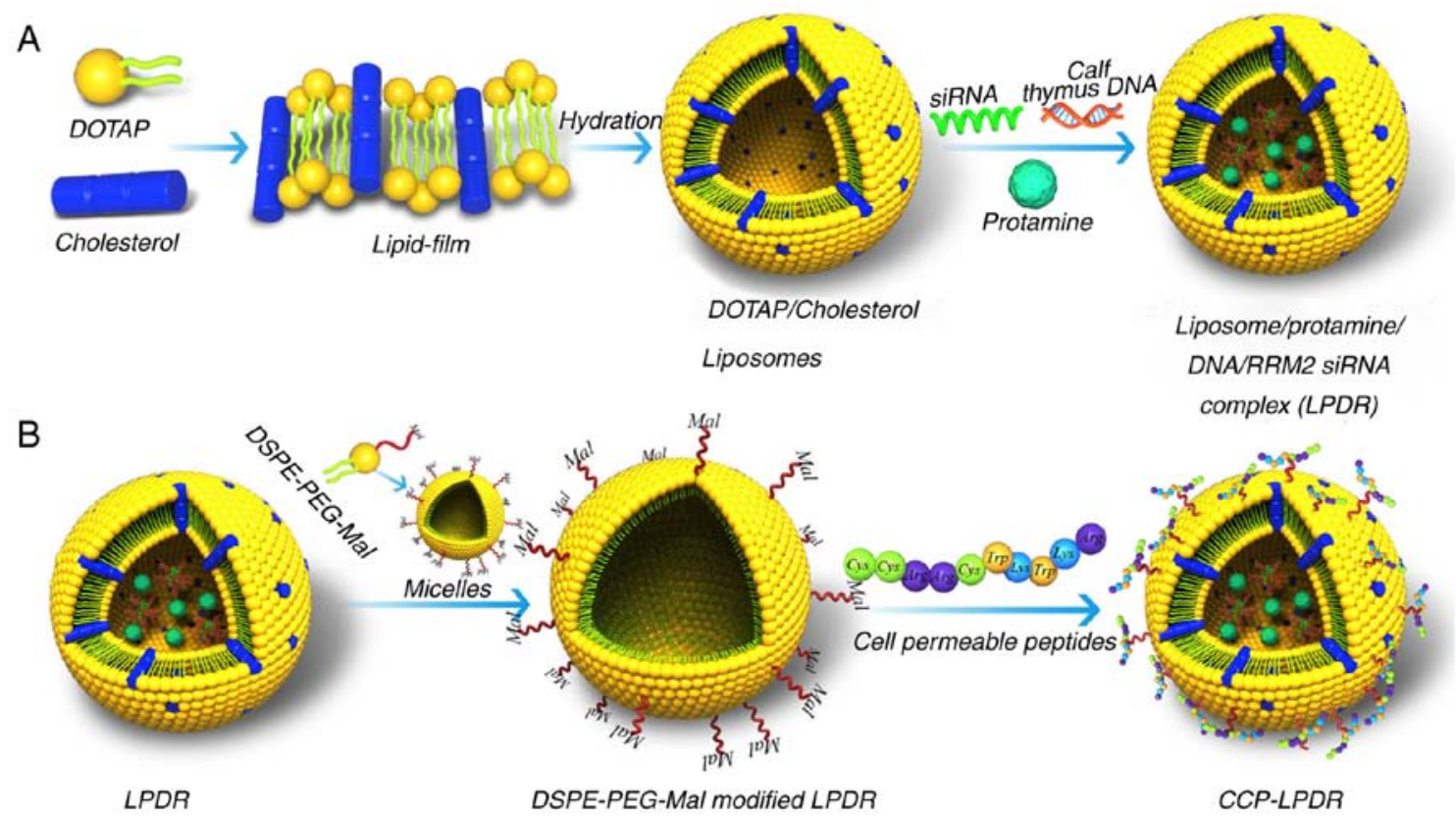

Figure 1. Development of cationic liposomes. (A) The development of cationic liposomes comprising DOTAP and cholesterol was performed using the thin film-based hydration approach. DOTAP/cholesterol liposomes were formed following hydration with water. Then, the LPDR was formed by mixing DOTAP/cholesterol liposomes, RRM2 siRNA, protamine and calf thymus DNA. (B) LPDR was inserted with the DSPE-PEG-Mal micelles, and CCP were conjugated with the DSPE-PEG-Mal modified LPDR to form CCP-LPDR. siRNA, small interfering RNA; CCP, cell permeable peptides; RRM2, ribonucleotide reductase M2; DOTAP, 1,2-dioleoyl-3-trimethylammonium-propane; DSPE-PEG-Mal, functional PEGylated lipids with a maleimide group, 1,2-distearoyl-sn-glycero-3-phosphoethanolamine-N-[maleimide (polyethylene glycol)-2000]; LPDR, liposome/protamine/DNA/RRM2 siRNA complex.

Table II. Characteristics of liposome-protamine-DNA-siRNA complex.

\begin{tabular}{lcccc}
\hline Liposomes & Size, $\mathrm{nm}$ & Zeta potential, $\mathrm{mv}$ & Polydispersity index & Encapsulation efficiency, \% \\
\hline CCP-LPDR & $135.32 \pm 9.35$ & $18.92 \pm 7.54$ & $0.15 \pm 0.06$ & $93.45 \pm 2.87$ \\
LPDR & $133.75 \pm 7.52$ & $22.63 \pm 7.36$ & $0.17 \pm 0.05$ & $94.29 \pm 1.34$ \\
CCP-LPDN & $142.82 \pm 8.13$ & $21.93 \pm 6.27$ & $0.18 \pm 0.03$ & $92.63 \pm 3.18$ \\
LPDN & $136.91 \pm 5.63$ & $20.34 \pm 5.63$ & $0.19 \pm 0.07$ & $90.98 \pm 5.25$ \\
\hline
\end{tabular}

Data are presented as mean \pm standard deviation $(n=5)$. CCP, cell permeable peptide; LPD, liposome-polycation-DNA complex; RRM2, ribonucleotide reductase M2; siRNA, small interfering RNA; CCP-LPDR, CCP-conjugated LPD loaded with RRM2 siRNA; LPDR, LPD loaded with RRM2 siRNA; CCP-LPDN, CCP-conjugated LPD loaded with negative control siRNA; LPDN, LPD loaded with negative control siRNA.

Data from the experiment to evaluate stability, in which the liposomes were incubated in different media (PBS, PBS with $10 \%$ FBS and PBS with $20 \%$ FBS) at $25^{\circ} \mathrm{C}$ for 5 days, is presented in Fig. 2. Although an increase of $10-20 \mathrm{~nm}$ in the size of the liposomes was observed when they were in media containing FBS, the stability of liposomes was markedly high during the entire incubation period, suggesting that the serum did not significantly affect the stability of liposomes.

In vitro binding of liposomes to $R A-F L S$. To confirm whether $\mathrm{CCP}$ increased the targeting rate of liposomes, fluorescent liposomes were incubated with RA-FLS. Fig. 3A indicates that FAM-siRNA CCP-LPDN exhibited a significantly increased transfection efficiency compared with FAM-siRNA LPDN in RA-FLS in the stability assay $(\mathrm{P}<0.05)$. With increases in time interval, the transfection efficiency of FAM-siRNA

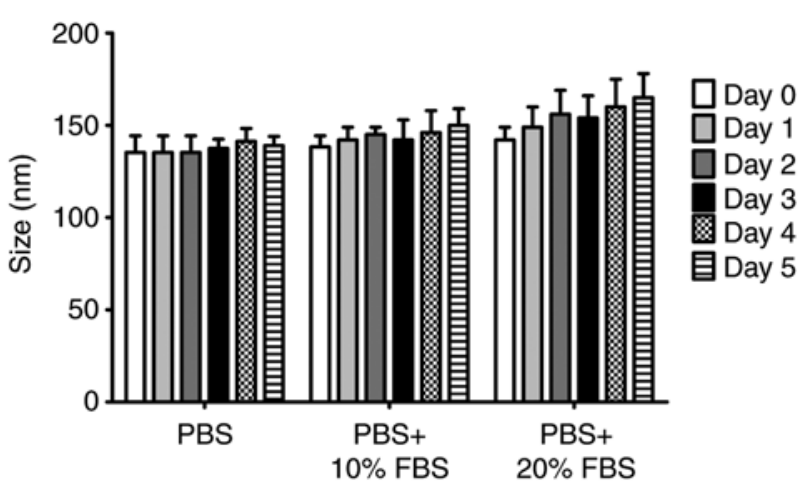

Figure 2. Stability of liposomes in different media. The liposomes were suspended in various media, including PBS, PBS with $10 \%$ FBS, or PBS with $20 \% \mathrm{FBS}$. Then, the solutions were incubated at $25^{\circ} \mathrm{C}$ for 5 days. On each day, an aliquot of liposomes was removed for analysis of the change in liposomal size. Data are presented as mean \pm standard deviation $(n=3)$. FBS, fetal bovine serum. 

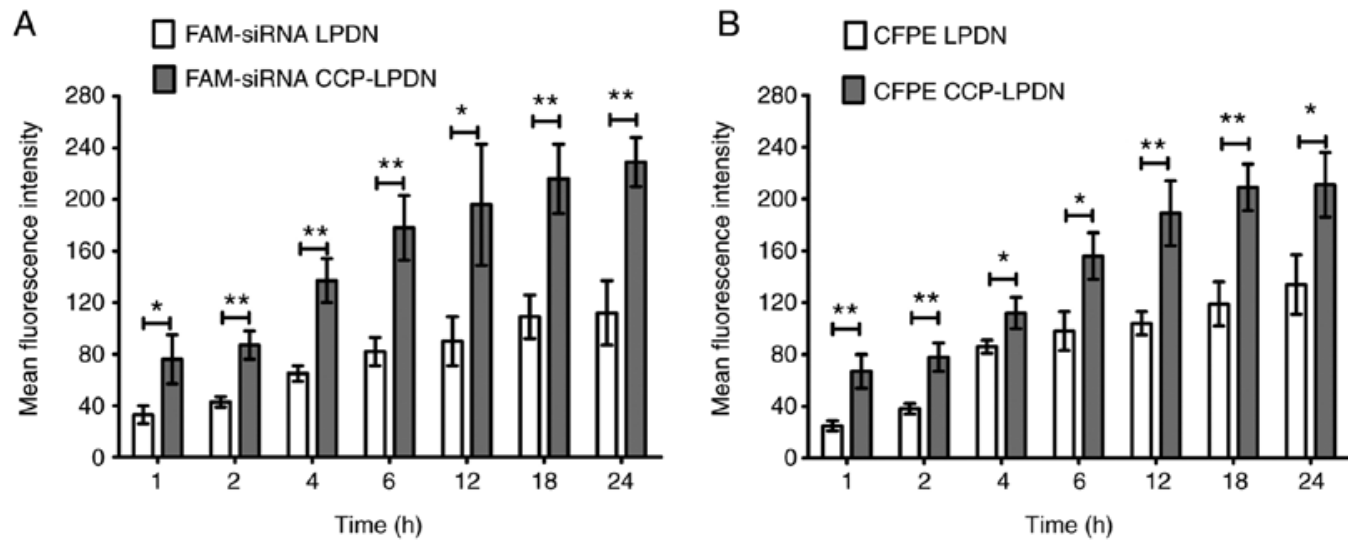

Figure 3. In vitro binding of liposomes in RA-FLS. RA-FLS were incubated with florescent liposomes (200 nM siRNA). Subsequently, the cells were washed to discard the liposomes. Finally, RA-FLS were trypsinized, washed and suspended in PBS. The analysis of the fluorescence was performed by flow cytometry. (A) FAM-siRNA loaded liposomes; (B) CFPE-labeled liposomes. " $\mathrm{P}<0.05 ;{ }^{* * *} \mathrm{P}<0.01$. Data are presented as mean \pm standard deviation ( $\mathrm{n}=3$ ). RA-FLS, rheumatoid arthritis fibroblast-like synoviocytes; CFPE, fluorescent lipid, 1,2-dioleoyl-sn-glycero-3-phosphoethanolamine-N-carboxyfluorescein; siRNA, small interfering RNA; FMA-siRNA, fluorescent siRNA; CCP, cell permeable peptides; LPD, liposome-polycation-DNA complex; LPDN, LPD loaded with negative control siRNA; CCP-LPDN, CCP-conjugated LPD loaded with negative control siRNA.
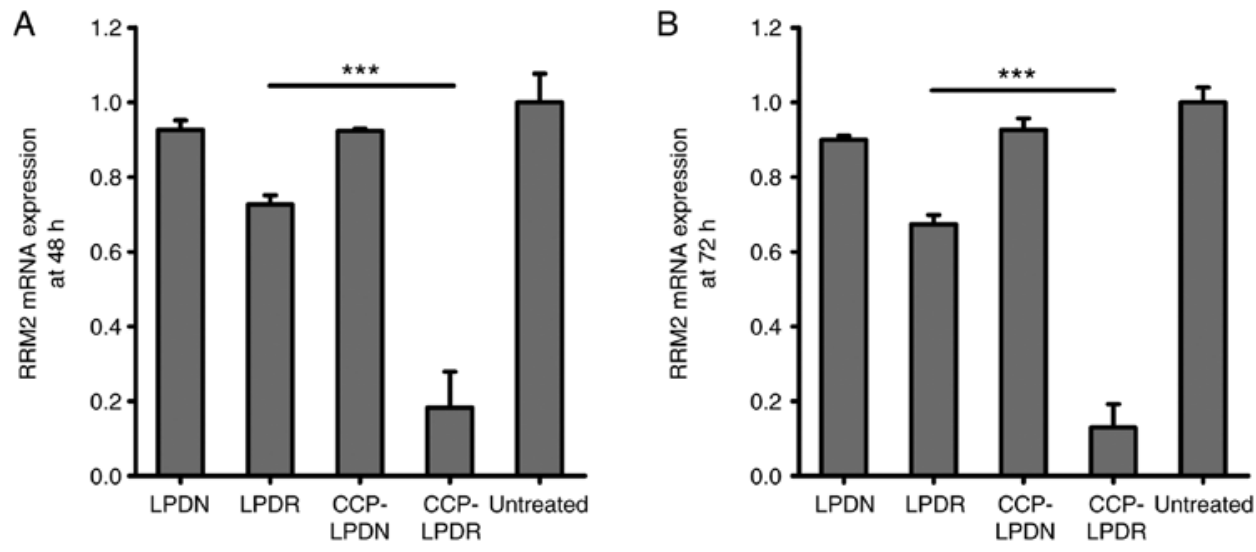

Figure 4. RT-qPCR-based analysis of RRM2 silencing. rheumatoid arthritis fibroblast-like synoviocytes were incubated with liposomes (200 nM siRNA) for $6 \mathrm{~h}$, and RT-qPCR was performed to analyze the RRM2 expression at (A) 48 and (B) $72 \mathrm{~h}$. The expression of RRM2 was normalized to $\beta$-actin. One-way analysis of variance with Student-Newman-Keuls post hoc test was used to compare the means among $\geq$ three groups. ${ }^{* * *} \mathrm{P}<0.001$. Data are presented as mean \pm standard deviation $(n=3)$. RT-qPCR, reverse transcription quantitative polymerase chain reaction; RRM2, ribonucleotide reductase M2; siRNA, small interfering RNA; LPD, liposome-polycation-DNA complex; LPDN, LPD loaded with negative control siRNA; CCP, cell permeable peptides; LPDR, LPD loaded with RRM2 siRNA.

LPDN also increased, and plateaued at $18 \mathrm{~h}$. Consistent with the FAM-siRNA assay, the in vitro binding of RA-FLS with CFPE-labeled liposomes exhibited similar results (Fig. 3B). CFPE CCP-LPND exhibited markedly increased transfection efficiency compared with CFPE LPND with RA-FLS $(\mathrm{P}<0.05)$. Again, with increase in time intervals, the transfection efficiency of CFPE CCP-LPND increased, and at $18 \mathrm{~h}$ reached a plateau.

Gene and protein silencing activity of RRM2 by liposomes in $R A-F L S$. The gene and protein silencing activity of RRM2 by liposomes was evaluated, and results are presented in Figs. 4 and 5. In the absence of RRM2 siRNA, LPDN and CCP-LPDN barely affected RRM2 gene expression (Fig. 4). LPDR exhibited poor gene silencing activity and inhibited the gene expression of RRM 2 by only $\sim 25 \%$. On the contrary, CCP-LPDR remarkably suppressed the RRM2 gene expression by $\sim 80 \%$, indicating an improved gene silencing activity compared with that exhibited by LPDR $(\mathrm{P}<0.001)$. The analysis of the expression of RRM2 protein obtained similar results, as demonstrated in Fig. 5. CCP-LPDR inhibited the expression of RRM2 protein by $\sim 80 \%$, whereas LPDR inhibited the expression of RRM2 protein by $\sim 20 \%(\mathrm{P}<0.001)$, indicating that the RRM2 suppression by CCP-LPDR was more efficient in comparison with LPDR. Therefore, it was confirmed that CCP-LPDR exhibited improved RRM2 suppression activity in RA-FLS in comparison with LPDR.

Activity of liposomes on the cellular proliferation and apoptosis in RA-FLS. The effect of liposomes on the levels of proliferation of RA-FLS was investigated using the CCK-8 assay (Fig. 6A). As demonstrated in Fig. 6A, LPDN and CCP-LPDN did not affect the proliferation of RA-FLS. It is noteworthy that CCP-LPDR exhibited significant inhibition of proliferation of RA-FLS compared with LPDR $(\mathrm{P}<0.001)$. Similarly, LPDN and CCP-LPDN did not induce significant apoptosis in RA-FLS, as reflected by the fact that apoptotic 
A
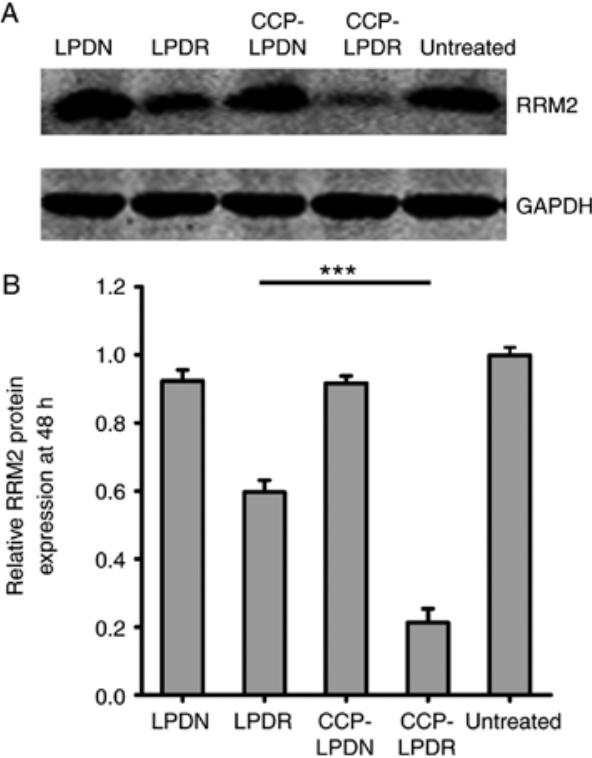

Figure 5. Western blot analysis. Rheumatoid arthritis fibroblast-like synoviocytes were incubated with liposomes ( $200 \mathrm{nM}$ siRNA) for $6 \mathrm{~h}$ and the analysis of RRM2 expression was performed at $72 \mathrm{~h}$. (A) One representative result of the western blot analysis. (B) The quantitative analysis of the western blot result. The RRM2 expression of the untreated group was normalized to its GAPDH expression, and the quantity of the RRM2 expression of the untreated group was defined as 1.0. One-way analysis of variance with Student-Newman-Keuls post hoc test was performed to compare the means among $\geq$ three groups. ${ }^{* * * *} \mathrm{P}<0.001$. Data are presented as mean \pm standard deviation $(n=3)$. RRM2, ribonucleotide reductase M2; siRNA, small interfering RNA; LPD, liposome-polycation-DNA complex; LPDN, LPD loaded with negative control siRNA; CCP, cell permeable peptides; LPDR, LPD loaded with RRM2 siRNA.

cells constituted only $\sim 5 \%$ of the total population of cells following their treatment, which was comparable to that observed in the untreated control group (Fig. 6B and C). However, the percentage of apoptotic cells was noticeably increased, and reached $\sim 15 \%$, following LPDR treatment, and CCP-LPDR treatment induced an even higher percentage of apoptotic cells $(\sim 30 \%)$ compared with LPDR $(\mathrm{P}<0.001)$, suggesting that CCP-LPDR exhibited the highest efficiency in inducing apoptosis in RA-FLS.

Analysis of proinflammatory cytokines in RA-FLS following liposome treatment. IL- 6 and TNF- $\alpha$ are potent proinflammatory cytokines secreted by RA-FLS, and they are pleiotropic cytokines with pivotal roles in the pathophysiology of RA $(25,26)$. They are used as valuable indexes to evaluate the efficacy of treatment of RA $(25,26)$. As indicated in Fig. 7, the levels of IL- 6 and TNF- $\alpha$ in the treated group were measured as the percentage of their mRNA and protein levels normalized to the untreated group (Fig. 7). LPDN and CCP-LPDN affected RRM2 expression levels minimally, whereas LPDR and CCP-LPDR significantly inhibited its expression in RA-FLS at mRNA and protein levels. As demonstrated in Fig. 7A, CCP-LPDR induced a more efficient inhibition of the TNF- $\alpha$ mRNA expression compared with CCP-LPDN $(\mathrm{P}<0.001)$ and LPDR $(\mathrm{P}<0.01)$. Similarly, CCP-LPDR was also more effective in inhibiting IL-6 mRNA expression compared with CCP-LPDN $(\mathrm{P}<0.001)$ and LPDR ( $\mathrm{P}<0.01)$ (Fig. 7B). With respect to the TNF- $\alpha$ and IL-6 protein levels, similar results were achieved (Fig. 7C and D). CCP-LPDR was more effective in inhibiting the TNF- $\alpha$ and IL- 6 protein levels compared with CCP-LPDN and LPDR $(\mathrm{P}<0.001)$, whereas LPDN and CCP-LPDN minimally affected the protein levels of TNF- $\alpha$ and IL- 6 in RA-FLS. Taken together, the expression levels of TNF- $\alpha$ and IL-6, the proinflammatory cytokines, were markedly decreased in RA-FLS following CCP-LPDR treatment.
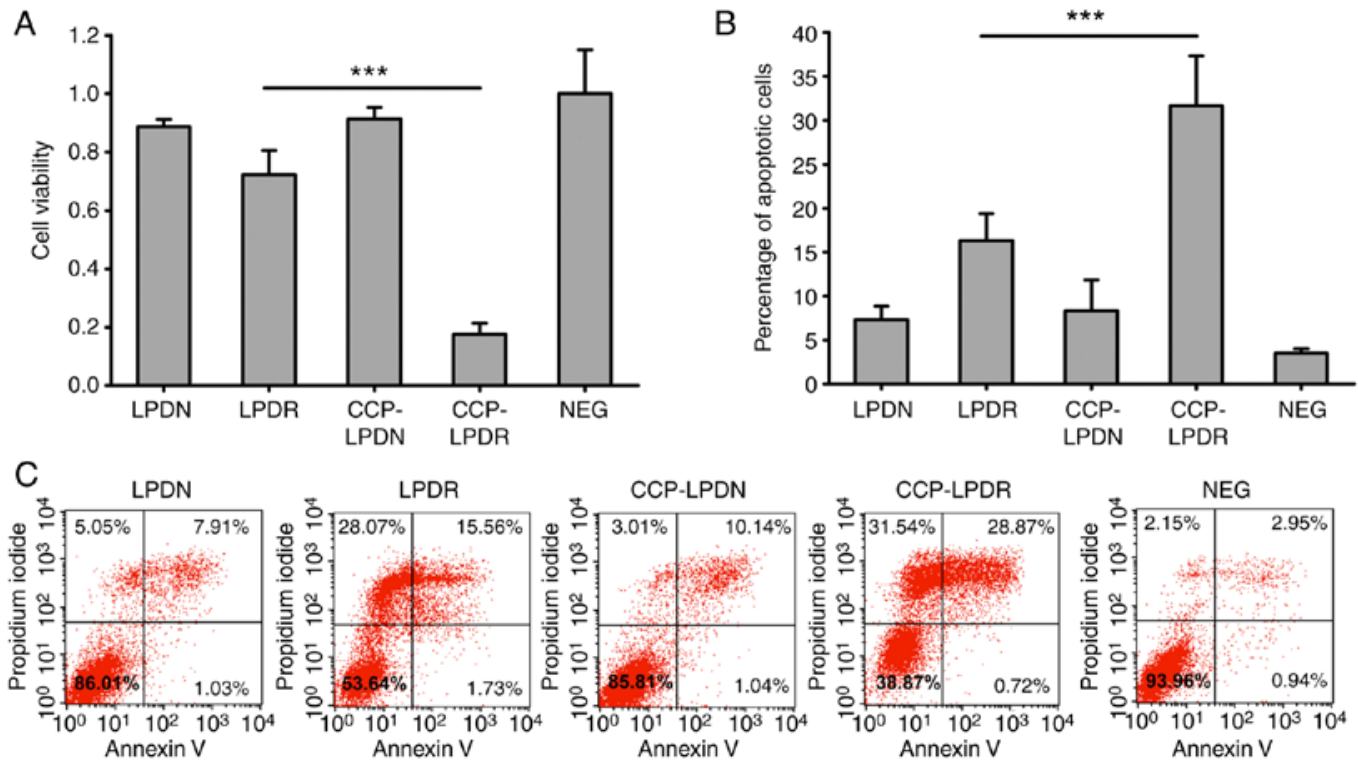

Figure 6. Analysis of cell viability and apoptosis. (A) Cell Counting Kit-8 assay. (B) Apoptosis was analyzed by the Alexa Fluor ${ }^{\circledR} 488$ Annexin V assay. One-way analysis of variance with Student-Newman-Keuls post hoc test was performed to compare the means among $\geq$ three groups. ${ }^{* * *} \mathrm{P}<0.001$. Data are presented as mean \pm standard deviation $(n=3)$. (C) One representative image of the apoptosis assay is presented. siRNA, small interfering RNA; LPD, liposome-polycation-DNA complex; LPDN, LPD loaded with negative control siRNA; CCP, cell permeable peptides; LPDR, LPD loaded with ribonucleotide reductase $\mathrm{M} 2$ siRNA; NEG, negative. 


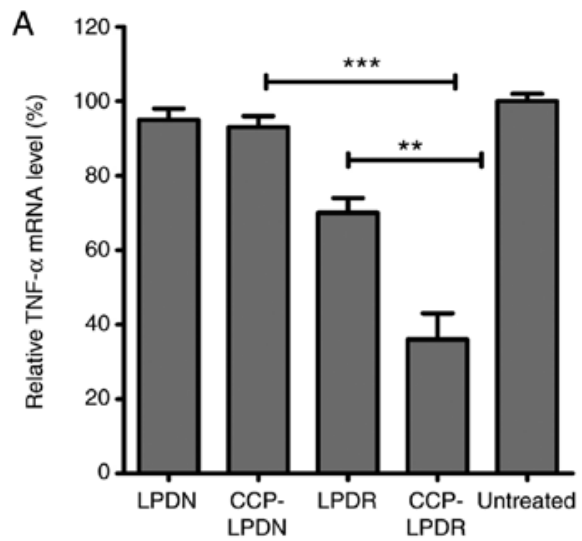

B
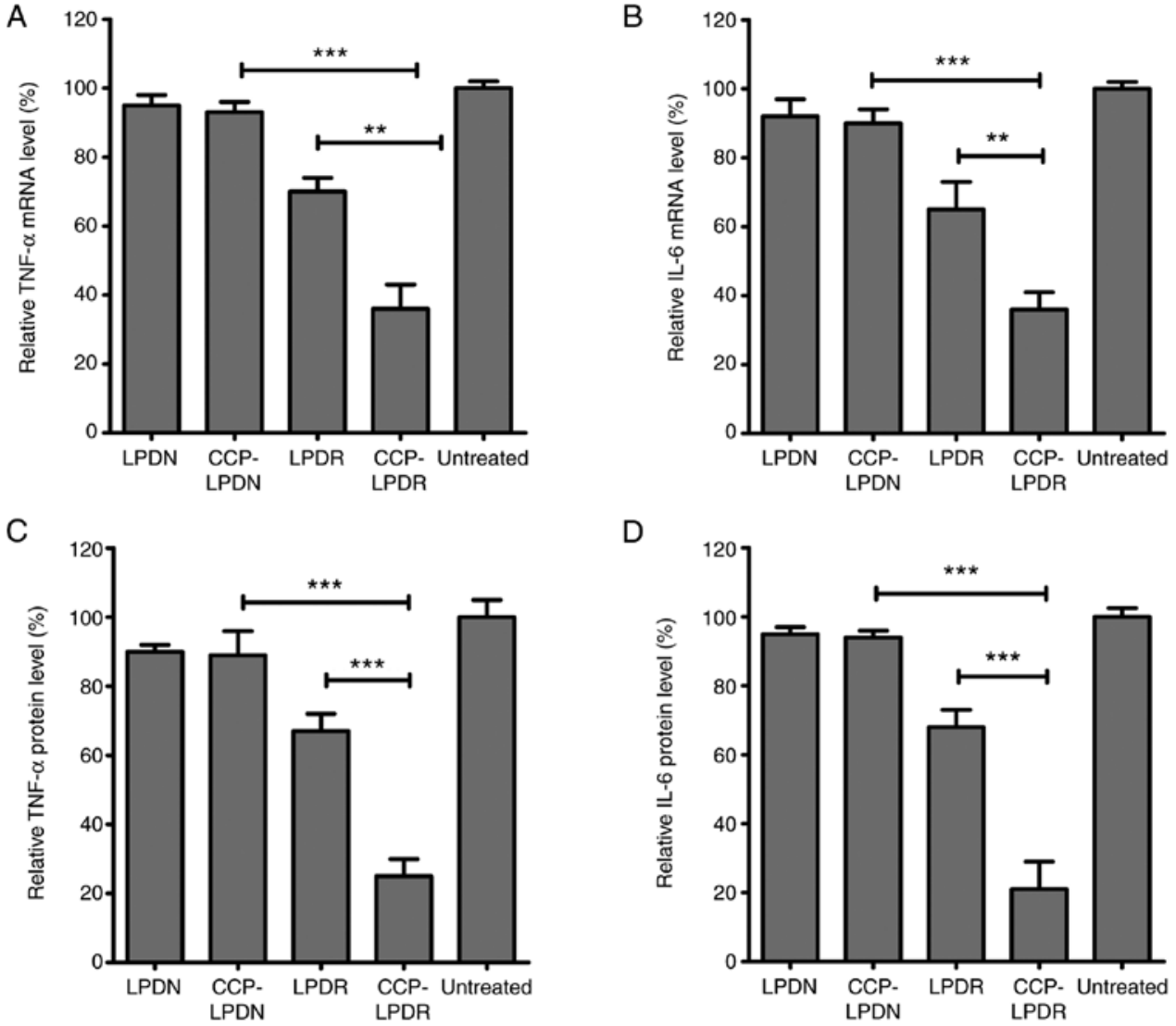

D

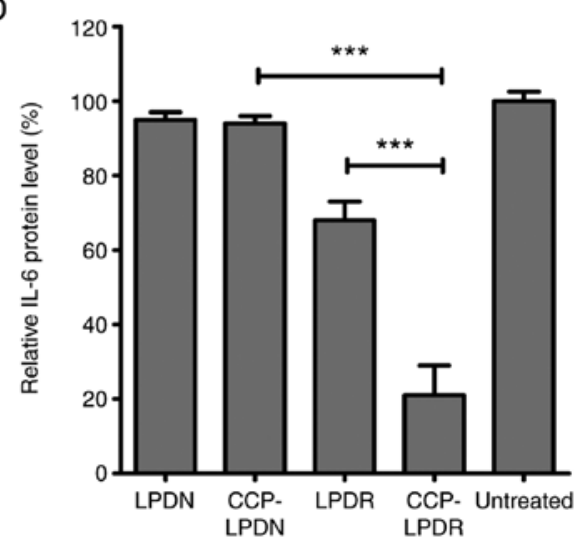

Figure 7. Analysis of proinflammatory cytokines in rheumatoid arthritis fibroblast-like synoviocytes following liposome treatment. (A) The relative TNF- $\alpha$ mRNA level. (B) The relative IL-6 mRNA level. (C) The relative TNF- $\alpha$ protein level. (D) The relative IL-6 protein level. The mRNA and protein levels of cytokines were expressed as the percentage of the protein of the treated groups normalized to the untreated group. ${ }^{* *} \mathrm{P}<0.01 ;{ }^{* * *} \mathrm{P}<0.001$. Data are presented as mean \pm standard deviation $(\mathrm{n}=3)$. TNF- $\alpha$, tumor necrosis factor $\alpha$; IL-6, interleukin-6; siRNA, small interfering RNA; LPD, liposome-polycation-DNA complex; LPDN, LPD loaded with negative control siRNA; CCP, cell permeable peptides; LPDR, LPD loaded with ribonucleotide reductase M2 siRNA.

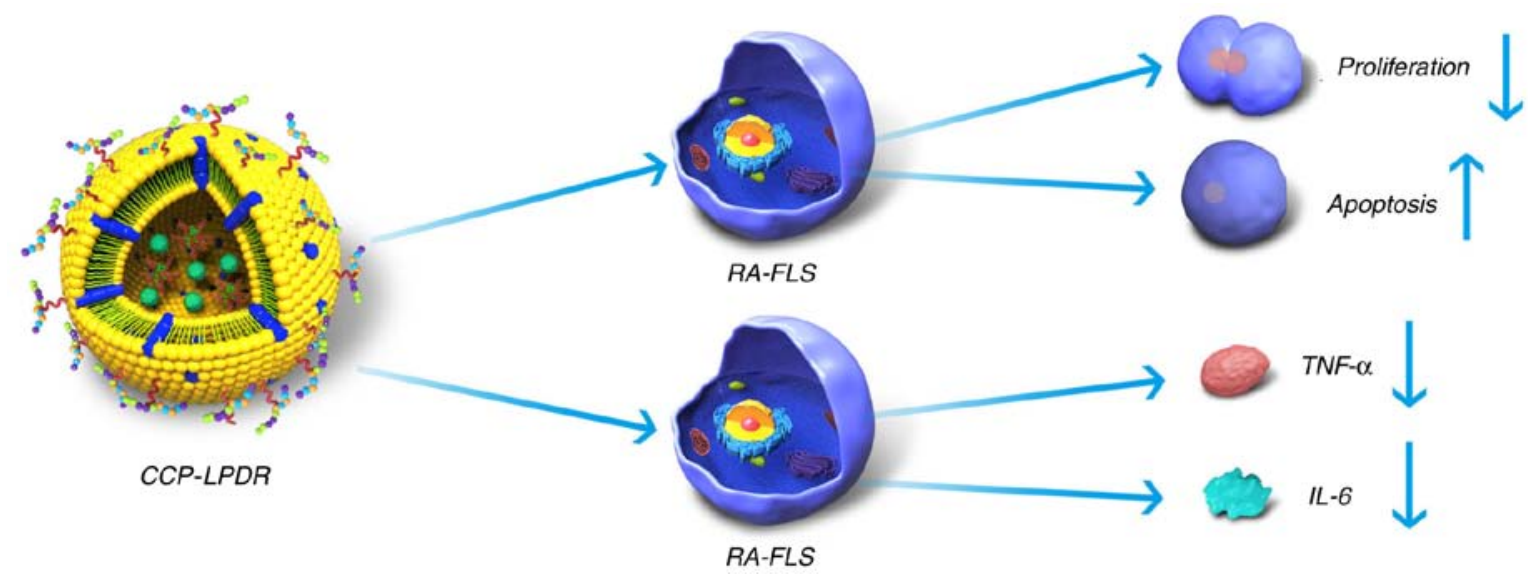

Figure 8. Mechanism underlying the anti-RA activity of LPDR. LPDR efficiently targeted RA-FLS, resulting in the proliferation inhibition and increased apoptosis of RA-FLS. LPDR also decreased the production of TNF- $\alpha$ and IL-6 in RA-FLS. RA-FLS, rheumatoid arthritis fibroblast-like synoviocytes; TNF- $\alpha$, tumor necrosis factor $\alpha$; IL-6, interleukin-6; CCP, cell permeable peptides; LPD, liposome-polycation-DNA complex; LPDR, LPD loaded with ribonucleotide reductase M2 small interfering RNA.

\section{Discussion}

RA incurs high costs of treatment with respect to surgical procedures, medications including biologics and indirect costs. Therefore, optimized treatments are required for the effective treatment of RA. Accumulating evidence has suggested that RA-FLS serve a key role in the progression of RA by promoting the production of proteases (6-8). The present study developed CCP-LPDR, which efficiently delivered RRM2 siRNA to RA-FLS, achieving an improved therapeutic efficacy against RA-FLS compared with the non-targeted control. 
The choice of target in gene therapy is crucial in liposome-based gene therapy. In the present study, RRM2 was selected as it is a crucial protein involved in DNA repair and synthesis $(11,12)$. Due to the substantial effect of RRM2 on the development and metastasis of tumors, RRM2 inhibitors (for example, GTI-2040 and gemcitabine) have been recruited for clinical trials for various types of cancer (13). Our previous study validated that RRM2 is a superior target for the treatment of liver cancer, and following RRM2 suppression, the levels of migration and proliferation of liver cancer cells were significantly inhibited (14). The progression of RA is similar to benign tumors, and the proliferation of RA-FLS and abnormal synovium promote the progression of RA (6). Therefore, the inhibition of RA-FLS proliferation was hypothesized to be a potential treatment for RA. Considering that RRM2 serves a crucial role in proliferation of RA-FLS, the present study aimed to inhibit their proliferation by suppressing RRM2 expression in RA-FLS. To the best of our knowledge, the present study is the first to demonstrate that, following RRM2 suppression in RA-FLS by CCP-LPDR, the proliferation of RA-FLS was significantly inhibited by $\sim 80 \%$ compared with the untreated control, and the level of apoptosis observed was $\sim 30 \%$ (Fig. 6). It is also the first study to confirm that, by RRM2 suppression via CCP-LPDR, significant inhibition of cellular proliferation and promotion in cellular apoptosis of RA-FLS may be induced, suggesting that RRM2 is a good therapeutic target for RA.

Developing improved siRNA-loaded nanoparticles is a critical step for the translation of siRNA-based therapies into clinical settings (24). Cationic liposomes serve as potential nanocarriers for siRNA delivery, however, they are prone to fast clearance following uptake by reticuloendothelial system (RES) (24). The attachment of opsonins to cationic liposomes is the primary reason for a high affinity of RES for unprotected cationic liposomes (25). Stealth liposomes with surface grafted PEG is a practical approach to decrease the RES uptake, by shielding the charge on the surface of the liposomes (26). In our previous studies investigating breast and liver cancer, the LPD complexes prepared were advanced PEGylated cationic liposomes, which exhibited a long circulation time in vivo and accumulation in the body (17-19). Therefore, the CCP-LPDR in the present study was expected to avoid RES uptake in vivo. It was demonstrated that the presence of CCP contributed significantly to the uptake of CCP-LPDR in RA-FLS. The present study indicated that the transfection efficiency of CCP-LPDR was markedly increased in comparison with LPDN, achieving marked inhibition of RRM2 gene and protein expression in RA-FLS. Subsequent to cell binding, CCP-LPDR induced an increased inhibition of proliferation and promotion of apoptosis in RA-FLS compared with LPDR, suggesting that CCP may significantly improve the targeting and treatment efficacy of CCP-LPDR in RA-FLS.

RA-FLS secrete IL-6 and TNF- $\alpha$, which are potent proinflammatory cytokines that promote the progression of RA $(27,28)$. It is noteworthy that the CCP-LPDR method was more effective in the inhibition of the TNF- $\alpha$ and IL- 6 protein levels in comparison with CCP-LPDN and LPDR, whereas LPDN and CCP-LPDN barely affected the protein levels of TNF- $\alpha$ and IL-6 in RA-FLS. Therefore, TNF- $\alpha$ and IL-6, the proinflammatory cytokines, were markedly decreased in RA-FLS following CCP-LPDR treatment.

As FLS are required to be obtained by primary culture from rats, a universal protocol of the culturing of primary FLS from rats has not been successfully established. Therefore, the effect of the treatment on normal FLS has not been assessed at present. Nevertheless, examining the effect of the treatment on normal FLS is important and will be incorporated into future study if possible.

Taken together, the present study suggested the mechanism of treatment efficacy of CCP-LPDR in RA (Fig. 8). Firstly, CCP-LPDR was targeted to and internalized by RA-FLS. In the cytoplasm, CCP-LPDR released RRM2 siRNA, achieving a combined therapeutic efficacy by increasing the inhibition of proliferation and promoting apoptosis in RA-FLS. Furthermore, the levels of proinflammatory cytokines TNF- $\alpha$ and IL-6 were also markedly decreased by the treatment with CCP-LPDR in RA-FLS.

The establishment of a potential treatment for RA-FLS cells is a viable approach for the treatment of RA. In the present study, a CCP-LPDR system was developed, which efficiently delivered RRM2 siRNA to RA-FLS, obtaining increased therapeutic efficacy with RA-FLS by increasing the levels of apoptosis and inhibition of cellular proliferation and proinflammatory cytokines. In conclusion, CCP-LPDR offered the possibility of suppressing RA-FLS and therefore provided a potential therapeutic approach for RA.

\section{Acknowledgements}

Not applicable.

\section{Funding}

The present study was funded by the National Natural Science Foundation of China (grant nos. 81771964 and 81472829).

\section{Availability of data and materials}

All data generated or analyzed during this study are included in this published article.

\section{Authors' contributions}

SF contributed to the design of the study and wrote the manuscript. XiW performed the experiments. XuW and JS analyzed the data. All authors have read and approved the final manuscript.

\section{Ethics approval and consent to participate}

Not applicable.

\section{Patient consent for publication}

Not applicable.

\section{Competing interests}

The authors declare that they have no competing interests. 


\section{Authors' information}

DrXin Wang, The First Department of Pain, Qingdao Municipal Hospital, Qingdao, Shandong 266011; Dr Xueping Wang and Dr Shiou Fu, The Second Department of Pain, Qingdao Municipal Hospital, Qingdao, Shandong 266011; Dr Jin Sun, International Joint Cancer Institute, Second Military Medical University, Shanghai 200433, P.R. China.

\section{References}

1. Mclnnes IB and Schett G: The pathogenesis of rheumatoid arthritis. N Engl J Med 365: 2205-2219, 2011.

2. Glant TT, Mikecz K and Rauch TA: Epigenetics in the pathogenesis of rheumatoid arthritis. BMC Med 12: 35, 2014.

3. Dougados M, Soubrier M, Antunez A, BalintP, Balsa A, Buch MH, Casado G, Detert J, El-Zorkany B, Emery P, et al: Prevalence of comorbidities in rheumatoid arthritis and evaluation of their monitoring: Results of an international, cross-sectional study (COMORA). Ann Rheum Dis 73: 62-68, 2014.

4. Gabriel SE, Crowson CS, Kremers HM, Doran MF, Turesson C, O'Fallon WM and Matteson EL: Survival in rheumatoid arthritis: A population-based analysis of trends over 40 years. Arthritis Rheum 48: 54-58, 2003.

5. Kobelt $G$ and Jönsson B: The burden of rheumatoid arthritis and access to treatment: outcome and cost-utility of treatments. Eur J Health Econ 8 (Suppl 2): S95-S106, 2008.

6. Guo Q, Wang Y, Xu D, Nossent J, Pavlos NJ and Xu J: Rheumatoid arthritis: Pathological mechanisms and modern pharmacologic therapies. Bone Res 6: 15, 2018.

7. Boechat Nde O, Ogusku MM, Boechat AL and Sadahiro A: Interaction between smoking and HLA-DRB1*04 gene is associated with a high cardiovascular risk in Brazilian Amazon patients with rheumatoid arthritis. PLoS One 7: e41588, 2012.

8. Bartok B and Firestein GS: Fibroblast-like synoviocytes: Key effector cells in rheumatoid arthritis. Immunol Rev 233: 233-255, 2010.

9. Konisti S, Kiriakidis S and Paleolog EM: Hypoxia - a key regulator of angiogenesis and inflammation in rheumatoid arthritis Nat Rev Rheumatol 8: 153-162, 2012.

10. Tang Q, Cui J, Tian Z, Sun J, Wang Z, Chang S and Zhu S: Oxygen and indocyanine green loaded phase-transition nanoparticle-mediated photo-sonodynamic cytotoxic effects on rheumatoid arthritis fibroblast-like synoviocytes. Int J Nanomedicine 12: 381-393, 2017.

11. Duxbury MS, Ito H, Zinner MJ, Ashley SW and Whang EE: RNA interference targeting the M2 subunit of ribonucleotide reductase enhances pancreatic adenocarcinoma chemosensitivity to gemcitabine. Oncogene 23: 1539-1548, 2004.

12. Zhang K, Hu S, Wu J, Chen L, Lu J, Wang X, Liu X, Zhou B and Yen Y: Overexpression of RRM2 decreases thrombspondin-1 and increases VEGF production in human cancer cells in vitro and in vivo: Implication of RRM2 in angiogenesis. Mol Cancer 8: $11,2009$.

13. Shao J, Zhou B, Chu B and Yen Y: Ribonucleotide reductase inhibitors and future drug design. Curr Cancer Drug Targets 6 : 409-431, 2006

14. Gao J, Chen H, Yu Y, Song J, Song H, Su X, Li W, Tong X, Qian W, Wang H, et al: Inhibition of hepatocellular carcinoma growth using immunoliposomes for co-delivery of adriamycin and ribonucleotide reductase M2 siRNA. Biomaterials 34 10084-10098, 2013.
15. Roman-Blas JA and Jimenez SA: NF-kappaB as a potential therapeutic target in osteoarthritis and rheumatoid arthritis. Osteoarthritis Cartilage 14: 839-848, 2006.

16. TomitaT,TakeuchiE,TomitaN,MorishitaR,KanekoM,YamamotoK, Nakase T, Seki H, Kato K, Kaneda Y, et al: Suppressed severity of collagen-induced arthritis by in vivo transfection of nuclear factor kappaB decoy oligodeoxynucleotides as a gene therapy. Arthritis Rheum 42: 2532-2542, 1999.

17. Gao J, Liu W, Xia Y, Li W, Sun J, Chen H, Li B, Zhang D, Qian W, Meng Y, et al: The promotion of siRNA delivery to breast cancer overexpressing epidermal growth factor receptor through anti-EGFR antibody conjugation by immunoliposomes. Biomaterials 32: 3459-3470, 2011.

18. Gao J, Yu Y, Zhang Y, Song J, Chen H, Li W, Qian W, Deng L, Kou G, Chen J, et al: EGFR-specific PEGylated immunoliposomes for active siRNA delivery in hepatocellular carcinoma. Biomaterials 33: 270-282, 2012.

19. Gao J, Sun J, Li H, Liu W, Zhang Y, Li B, Qian W, Wang H, Chen J and Guo Y: Lyophilized HER2-specific PEGylated immunoliposomes for active siRNA gene silencing. Biomaterials 31: 2655-2664, 2010.

20. Xu C, Lee SA and Chen X: RNA interference as therapeutics for hepatocellular carcinoma. Recent Pat Anticancer Drug Discov 6: 106-115, 2011.

21. Gao H, Zhang Q, Yu Z and He Q: Cell-penetrating peptide-based intelligent liposomal systems for enhanced drug delivery. Curr Pharm Biotechnol 15: 210-219, 2014.

22. Koren E and Torchilin VP: Cell-penetrating peptides: Breaking through to the other side. Trends Mol Med 18: 385-393, 2012.

23. Jha D, Mishra R, Gottschalk S, Wiesmüller KH, Ugurbil K, Maier ME and Engelmann J: CyLoP-1: A novel cysteine-rich cell-penetrating peptide for cytosolic delivery of cargoes. Bioconjug Chem 22: 319-328, 2011.

24. Livak KJ and Schmittgen TD: Analysis of relative gene expression data using real-time quantitative PCR and the $2^{-\Delta \Delta C_{\mathrm{T}}}$ method. Methods 25: 402-408, 2001.

25. Srirangan S and Choy EH: The role of interleukin-6 in the pathophysiology of rheumatoid arthritis. Ther Adv Musculoskelet Dis 2: $247-256,2010$.

26. Alonso-Ruiz A, Pijoan JI, Ansuategui E, Urkaregi A, Calabozo M and Quintana A: Tumor necrosis factor alpha drugs in rheumatoid arthritis: Systematic review and metaanalysis of efficacy and safety. BMC Musculoskelet Disord 9: 52, 2008.

27. Lee H,Lytton-Jean AK, Chen Y,Love KT,Park AI,KaragiannisED, Sehgal A, Querbes W, Zurenko CS, Jayaraman M, et al: Molecularly self-assembled nucleic acid nanoparticles for targeted in vivo siRNA delivery. Nat Nanotechnol 7: 389-393, 2012.

28. Tseng YC, Mozumdar S and Huang L: Lipid-based systemic delivery of siRNA. Adv Drug Deliv Rev 61: 721-731, 2009.

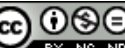

This work is licensed under a Creative Commons Attribution-NonCommercial-NoDerivatives 4.0 International (CC BY-NC-ND 4.0) License. 